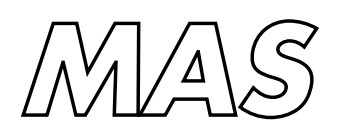

Modelling, Analysis and Simulation Modelling, Analysis and Simulation
MAS Numerical convergence of the branching time of
negative streamers

C. Montijn, U. Ebert, W. Hundsdorfer

RePORT MAS-E0613 APRIL 2006 
Centrum voor Wiskunde en Informatica (CWI) is the national research institute for Mathematics and Computer Science. It is sponsored by the Netherlands Organisation for Scientific Research (NWO).

CWI is a founding member of ERCIM, the European Research Consortium for Informatics and Mathematics.

CWI's research has a theme-oriented structure and is grouped into four clusters. Listed below are the names of the clusters and in parentheses their acronyms.

Probability, Networks and Algorithms (PNA)

Software Engineering (SEN)

\section{Modelling, Analysis and Simulation (MAS)}

Information Systems (INS)

Copyright (C) 2006, Stichting Centrum voor Wiskunde en Informatica

P.O. Box 94079, 1090 GB Amsterdam (NL)

Kruislaan 413, 1098 SJ Amsterdam (NL)

Telephone +31205929333

Telefax +31205924199

ISSN 1386-3703 


\title{
Numerical convergence of the branching time of negative streamers
}

\begin{abstract}
In sufficiently large gaps and electric fields, discharge streamers do branch. In [Arrayás et al., PRL 88, 174502 (2002)], we observed streamer branching numerically within a deterministic particle density model and explained it as a Laplacian instability of a thin space charge layer. Our numerical results were criticized in [Kulikovsky, PRL 89, 229401 (2002)]. We here present an adaptive grid refinement method for streamer simulations, and we carry out the first conclusive investigation on the effect of the numerical grid on streamer branching in different fields. On stepwise finer grids the branching time converges, hence streamer branching is for the first time predicted quantitatively.
\end{abstract}

2000 Mathematics Subject Classification: 35k57, 65m50, 78a35

Keywords and Phrases: streamers, branching, adaptive grids, numerical convergence

Note: This work was carried out within theme MAS3. C.M.Ithanks the Netherlands Organization for Scientific Research (NWO) for a PhDIgrant within the programme on Computational Science. The paper was submitted to Phys. Rev. E. 



\title{
Numerical convergence of the branching time of negative streamers
}

\author{
Carolynne Montijn ${ }^{1}$, Ute Ebert ${ }^{1,2}$, and Willem Hundsdorfer ${ }^{1}$ \\ ${ }^{1}$ CWI, P.O.Box 94079, 1090 GB Amsterdam, The Netherlands, and \\ ${ }^{2}$ Dept. Physics, Eindhoven Univ. Techn., The Netherlands.
}

(Dated: April 3, 2006)

\begin{abstract}
In sufficiently large gaps and electric fields, discharge streamers do branch. In [Arrayás et al., PRL 88, 174502 (2002)], we observed streamer branching numerically within a deterministic particle density model and explained it as a Laplacian instability of a thin space charge layer. Our numerical results were criticized in [Kulikovsky, PRL 89, 229401 (2002)]. We here present an adaptive grid refinement method for streamer simulations, and we carry out the first conclusive investigation on the effect of the numerical grid on streamer branching in different fields. On stepwise finer grids the branching time converges, hence streamer branching is for the first time predicted quantitatively.
\end{abstract}

Problem setting and review. Streamers are transient weakly ionized plasma channels that rapidly grow into a non- or weakly ionized medium under influence of the self-enhanced electric field at their tip. They are widely used in technology [1, 2] and ubiquitous in nature, where they play a role in creating the path of sparks, lightning [3] and of blue jets above thunderclouds. Streamers are also directly observed as so-called sprites [4, 5, 6], which are very large discharge structures in the higher parts of the atmosphere that are composed of ten thousands of streamers. Despite their high velocity, streamer evolution is now directly observable in experiments; a further review can be found in [2].

Streamers commonly branch in experiments if gap and applied voltage are large enough. Recently a debate has risen about the proper physical concept for this branching. In 1939, Raether [7] proposed a mechanism for streamer propagation and Loeb and Meek [8] developed it into a branching concept that nowadays is found in many textbooks. The concept is based on a uniformly charged streamer head; ahead of it stochastic processes create secondary avalanches, that subsequently develop into different branches. However, the distribution of rare electrons due to photo-ionization or background ionization ahead of the streamer has never been shown to agree with the conceptual pictures, and the concept has never been demonstrated to work. Furthermore, simulations in the past two decades [9, 10, 11, 12] have shown that the fully developed streamer head is not homogeneously charged, but rather neutral and surrounded by a thin space charge layer which enhances the field ahead of it and screens it in the interior; this field enhancement allows the streamer to penetrate regions with a rather low background field. Recent simulations also show that a streamer can branch within a fully deterministic model for charged particle densities, in a non-uniform background field 13, 14, 15. as well as in a uniform field [16, 17, 18], provided certain requirements on the external parameters are met (e.g. a sufficiently strong background electric field and a sufficiently long gap).

Some of the present authors have proposed 16, 17] a physical explanation of these numerical observations that is directly related to the formation of the thin space charge layer: the layer creates an almost equipotential streamer head that can undergo a Laplacian instability and branch in a manner similar to branching instabilities of fluid interfaces in viscous fingering. For a further discussion of the conceptual questions of streamer branching, we refer to 2]. However, the numerical codes used in 13, 14, 15, 16, 17, 18] were not able to test the branching conditions on fine numerical grids. This lead some researchers to question the physical nature of the instabilities [14, 15, 19, 20] despite the analytical arguments given in [16, 17] and later in [21, 22].

To resolve the debate from the numerical side, we have developed a code with comoving adaptive grids and we here present its results. The algorithm enables us to run the simulations on very fine grids; therefore for the first time the effect of numerical grids on the branching process is investigated quantitatively. We here present its results: branching occurs both at very high fields like in Refs. 16, 17] and also at fairly low background fields if the discharge has sufficient space to develop; and the branching time saturates on sufficiently fine numerical grids. This enables us to give the first quantitative predictions on streamer branching.

Model and multiscale structure of negative streamers. We investigate a minimal continuum model for streamers, which contains the essential physics for negative streamers in a non-attaching pure gas like $\mathrm{N}_{2}$ or $\operatorname{Ar}[$ 9, 10, 16, 17]. The model is a two-fluid approximation for the charged particles, with a local field dependent impact ionization reaction coupled to the Poisson equation for electrostatic particle interactions. We investigate this model in a cylindrically symmetric geometry, reducing it to effectively two dimensions. This constraint suppresses one degree of freedom for the instability modes, and therefore the time of branching in this cylindrical geometry is an upper bound for the branching time in a genuine three dimensional system [2, 23]. In dimensionless units, the model reads

$$
\begin{aligned}
\partial_{\tau} \sigma & =\nabla \cdot(\sigma \mathcal{E}+D \nabla \sigma)+\sigma|\mathcal{E}| \alpha(|\mathcal{E}|), \\
\partial_{\tau} \rho & =\sigma|\mathcal{E}| \alpha(|\mathcal{E}|), \quad \alpha(|\mathcal{E}|)=e^{-1 /|\mathcal{E}|}, \\
-\nabla^{2} \phi & =\rho-\sigma, \quad \mathcal{E}=-\nabla \phi
\end{aligned}
$$

where $\sigma$ and $\rho$ are the electron and positive ion densities, 
respectively. $\mathcal{E}$ and $\phi$ are respectively the electric field and potential, $D$ is the electron diffusion coefficient and $\tau$ is the dimensionless time. The characteristic scales in this model depend on the neutral gas density; therefore the simulation results can be applied to high altitude sprite discharges at low pressures as well as to high pressure laboratory experiments. We refer to [2, 17, 24] for more details on the dimensional analysis.

A planar cathode is placed at $z=0$ and a planar anode at $z=L_{z}$. The potential at the electrodes is fixed, $\phi(r, z=0, \tau)=0, \phi\left(r, z=L_{z}, \tau\right)=\phi_{0}>0$, generating a background electric field with strength $\left|\mathcal{E}_{b}\right|=\phi_{0} / L_{z}$ along the negative $z$-direction. The streamer is initiated by an electrically neutral Gaussian ionization seed on the axis of symmetry at the cathode $(r=z=0)$. There is no background ionization far from the initial seed.

We impose homogeneous Neumann conditions for the electron density at all boundaries. This results in a net inflow of electrons from the cathode if the streamer is attached to it [16, 25]. In practice, the computational volume is restricted in the radial direction by a boundary $L_{r}$ sufficiently far away not to disturb the solution near and in the streamer. Moreover, we choose the interelectrode distance $L_{z}$ so large that the streamer does not feel the anode proximity for the results shown.

The generic spatial structure of the streamer is already discussed above and can be seen in the figures: it contains a wide range of spatial scales, from the very extended non-ionized medium on which the Poisson equation has to be solved through the length of the conducting channel and its width up to the inner structure of the thin space charge layer around the streamer head.

Moreover, the region just ahead of the streamer where the field is substantially enhanced and the electron density is low, is highly unstable, in the sense that a small ionized perturbation will grow much more rapidly than in the mere background field. This unstable region ahead of the streamer tip is commonly referred to as leading edge [24, 26]. It requires special care when considering numerical methods [25, 26]. Accurate simulations of streamers therefore pose a great computational challenge.

Numerical algorithm. In order to deal efficiently with the numerical challenges posed by this model, it has been implemented in a numerical code using adaptive grid refinements. We recall the essential features of this algorithm and refer to [25] for further details. The spatial discretizations are based on finite volumes, using a flux limiting scheme to prevent spurious oscillations in the results near steep gradients. The time stepping is performed with a two-stage explicit Runge-Kutta method.

Using an explicit time-stepping method allows us to decouple the computational grids for the continuity equations (11)-(2) on the one hand from those for the Poisson equation (3) on the other hand. The particle densities are first updated on a series of nested, stepwise refined grids. Then the Poisson equation, using the computed densities as an input, is solved on another series of nested, step-
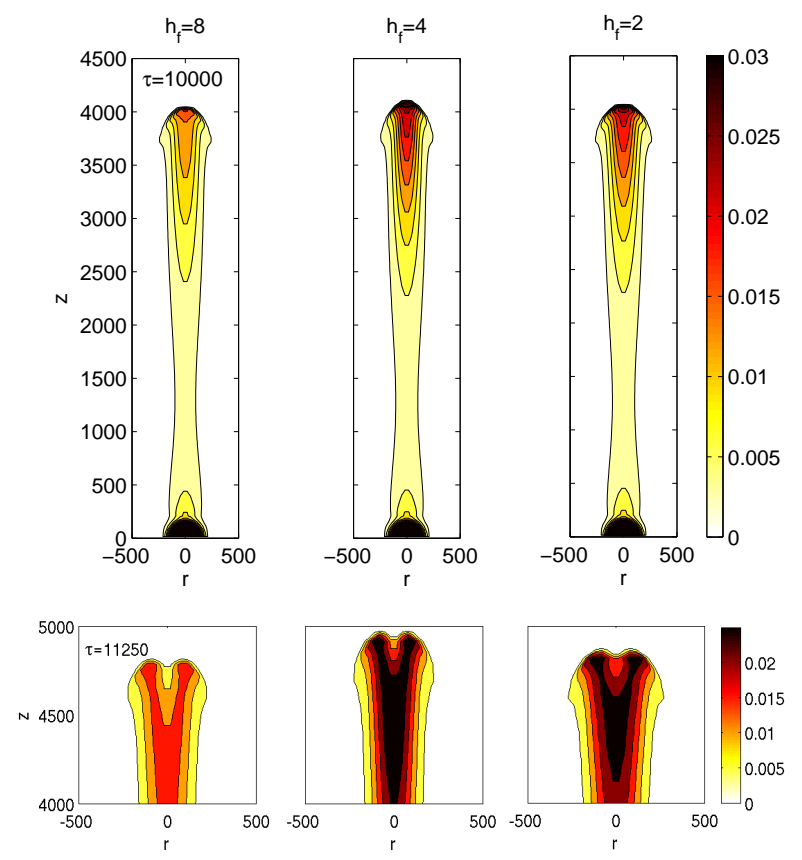

FIG. 1: Electron density distribution before and just after streamer branching in a background field $\left|\mathcal{E}_{b}\right|=0.15$, computed on different finest mesh sizes $h_{f}=8,4$ and 2 as indicated over the plots. The upper snapshots at $\tau=10000$ are taken before branching and the lower ones after branching, at time $\tau=11250$. The contours correspond to the same density levels. In all three cases the same restricted part of the total computational domain with $z \leq L_{z}=32768$ and $r \leq L_{r}=L_{z} / 2$ is shown.

wise refined grids. The electric field on the grids for the continuity equations is then calculated from the potential computed on the grids for the Poisson equation using sufficiently accurate interpolations [27].

Adequate refinement criteria for the continuity and for the Poisson equation then lead to a grid distribution which is especially designed to cope adequately and efficiently with the difficulties inherent to both type of equations. More specifically, the refinement criterion for the grids for the Poisson equation is based on error estimate of the solution. The refinement criterion for grids for the continuity equations uses a curvature monitor of the solution. Moreover, it takes explicitly into account the leading edge, where the densities are low but the electric field is greatly enhanced [24, 26].

The refinement criterion is computed at each time step, in such a way that the series of nested, consecutively refined grids move with the solution. Special care has been taken for the discretizations as well as the mapping of the solution from one grid to the other to be charge conserving.

Results. The adaptive grid refinement procedure enables us to resolve the streamer with very high accuracy, and thus to investigate the dependence of the branching 

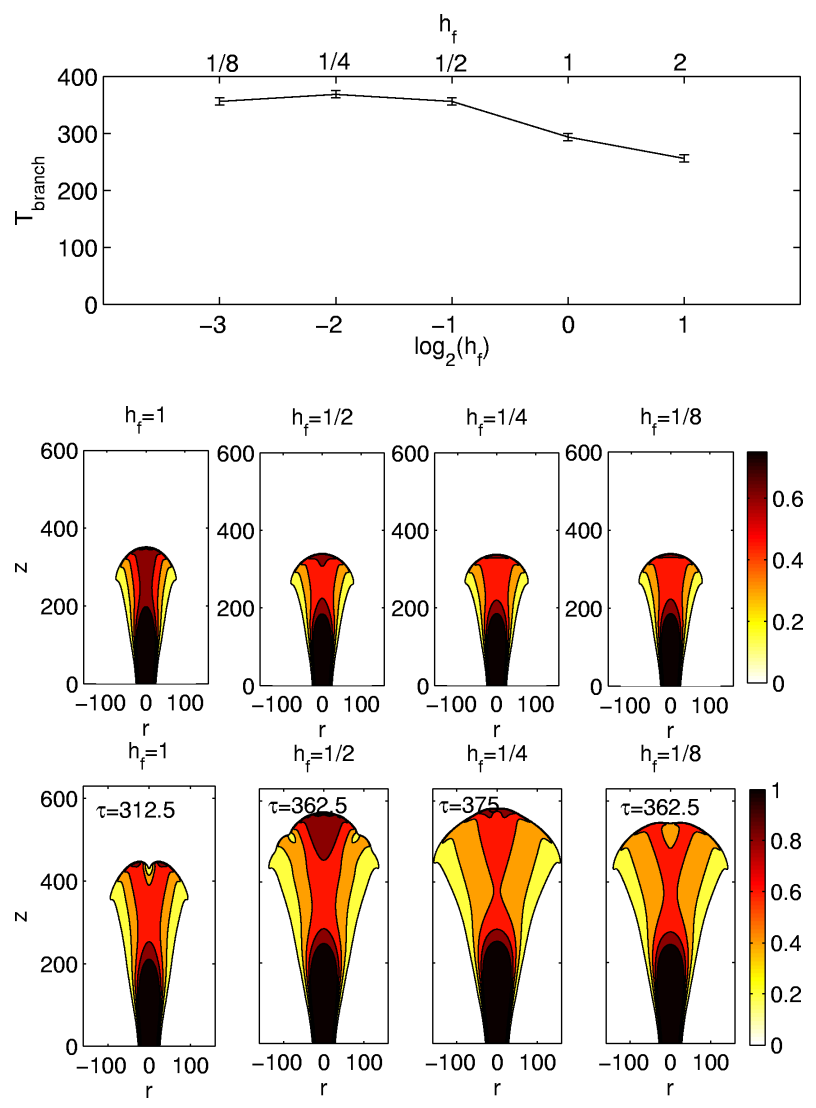

FIG. 2: Upper panel: Branching time in a background field $\left|\mathcal{E}_{b}\right|=0.5$ as function of the finest mesh size $h_{f}=2,1,1 / 2$, $1 / 4,1 / 8$. Lower panels: the corresponding electron density distribution at $\tau=275$ (middle row), and just after the respective branching time (lower row), computed on different finest grids $h_{f}=1,1 / 2,1 / 4$ and $1 / 8$. The total computational domain is $z \leq L_{z}=2048$ and $r \leq L_{r}=L_{z} / 2$.

process on the numerical grid. The results are obtained on increasingly finer grid sizes $h_{f}$, always taking the same coarsest mesh width $h_{c}$ for both the continuity and the Poisson equations. If the branching were of numerical nature, we would expect that branching times on increasingly finer grids would not converge.

We first consider negative streamers evolving in a low background field of $\mathcal{E}_{b}=0.15$ corresponding to $30 \mathrm{kV} / \mathrm{cm}$ for $\mathrm{N}_{2}$ at atmospheric pressure. We use an electrically neutral, dense and relatively wide Gaussian ionization seed at the cathode, with a maximum of $1 / 4.8$, and a characteristic radius of 10 . This corresponds to a maximal electron and ion number density of $10^{14} \mathrm{~cm}^{-3}$ and an $1 /$ e radius of $230 \mu \mathrm{m}$. The gap length and width are set to $L_{z}=2 L_{r}=2^{15}=32768$, which corresponds to an inter-electrode distance of approximately $7.5 \mathrm{~cm}$.

The coarsest mesh width is set to $h_{c}=64$, and the finest one to $h_{f}=8,4$ and 2 . When a finest mesh of 8 is used, the electron density in the streamer is lower, as can be seen in the upper row in Fig. 11 This is due to

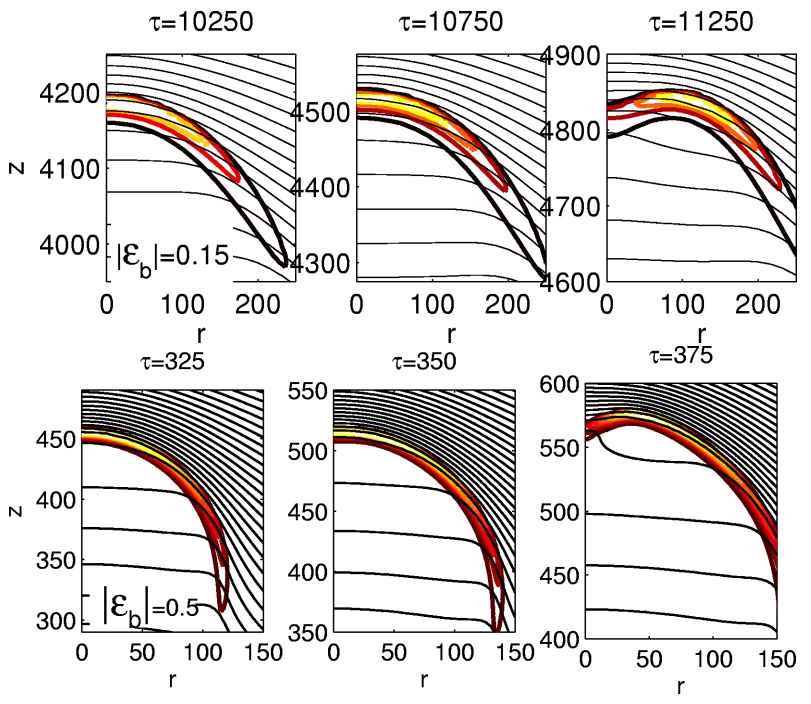

FIG. 3: Zoom into the streamer head during branching. Upper plots: $\left|\mathcal{E}_{b}\right|=0.15$ as in Fig. 1] $h_{f}=2$. Lower plots: $\left|\mathcal{E}_{b}\right|=0.5$ as in Fig. $2 h_{f}=1 / 8$. Contour lines (thick) of net charge density and equipotential lines (thin) are shown as a function of positive radius $r$ and appropriate $z$. The spacing of the charge contour levels is 0.004 for the low field case, and 0.16 in the high field case. The spacing of equipotential lines is 5 in both cases.

the numerical diffusion introduced on such a coarse grid by the flux limiter that switches to the diffusive first order scheme in regions with large gradients. This numerical diffusion smears the electrons out over the streamer head, which in turn results in lower field enhancement and lower ionization rates. The results on finer meshes of 4 and 2 on the other hand do agree with each other.

The branching in time is the same in all cases. Fig. 1] shows that the influence of the numerical grid on the branching state rapidly decreases, and we thus can carry out not only qualitative but also quantitative numerical experiments of the streamer evolution up to branching. These results show that branching is possible at lower electric fields than those of [16, 17]. Branching was not observed in earlier simulations at lower fields 9, 10] because the discharge gap was too short.

We now consider a negative streamer in a dimensionless background field of $\mathcal{E}_{b}=0.5$ corresponding to $100 \mathrm{kV} / \mathrm{cm}$ in $\mathrm{N}_{2}$ at atmospheric pressure in a gap of $L_{z}=2048$, or $4.6 \mathrm{~mm}$. These external parameters are as in [16, 17]. The initial seed is also taken as in [17], i.e., a Gaussian with amplitude $10^{-4}$ and characteristic radius 10 , which corresponds to a maximal electron and ion number density of $5 \cdot 10^{10} \mathrm{~cm}^{-3}$ and an $1 /$ e-radius of $23 \mu \mathrm{m}$ for $\mathrm{N}_{2}$ under normal conditions.

However, while [16] used a uniform grid of $h=2$ and [17] one of $h=1$, we now perform computations on a finest grid as small as $h_{f}=1 / 8$, i.e., more than a decade finer. More precisely, the coarsest mesh width is set to 
$h_{c}=2$, and the finest one to $h_{f}=2,1, \ldots, 1 / 8$. Furthermore a better numerical scheme is used: flux limiting [25] rather than 3rd order upwind [16, 17].

Before branching, at $\tau=275$, Fig. 2 shows that there is a quantitative difference between the results on a mesh with $h_{f}=1$ and the other three. As in the low field case, numerical diffusion spreads the space charge layer, which makes the field enhancement at the streamer tip and the field screening in the streamer body less efficient. Consequently, the ionization rate, and therefore the electron density, are higher in the streamer body. In the low field case we do not observe this because the background field is negligibly low, hence a less efficient screening will not affect significantly the ionization rate in the streamer body. It is clear that on meshes finer than $1 / 2$, the results are the same during the stable streamer propagation. It is only after the branching that different states are observed on those very fine grids. However, the time of branching converges within this range of mesh widths $h_{f}$ as shown in the upper plot in Fig. [2]

Discussion, conclusion and outlook. We emphasize that the branching times converge on decreasing numerical grids in both cases. Therefore we here present the first conclusive and quantitative numerical predictions on streamer branching. However, in contrast to the low field case, the lower plots in Fig. 2 show that in the high field case different branched modes are reached after approximately the same evolution time: in two cases, the maximal electron density and field is on the axis of symmetry, and in two other cases, it is off axis. Apparently, there are different branched states reachable at bifurcation and tiny differences determine which one will be reached. Such extreme sensitivity is well-known from deterministic chaos; it is generic for nonlinear dynamics near bifurcation points. On the other hand, the unstable state is reached in a deterministic manner, and therefore the branching times converge.

But why is there once a unique branched state and once several? The answer can be found in Fig. 3] showing the two relevant spatial scales, namely the thickness of the space charge layer and the radius of the channel. In the high field case, the ratio of layer thickness over radius is much smaller than in the low field case. Moreover, the field screening and enhancement is much stronger and the equipotential lines follow the space charge layer much better. Therefore the high field streamer is much closer to interfacial models as discussed in 2, 16, 21, 22, 24] and can access more branching modes. This critical state in future work will be characterized by the electric charge content and electric field and potential at the streamer tip which would then allow us to relate branching to the external electric circuit. For sketches of such ideas as well as for a discussion of photo-ionization effects and of continuum versus particle models, we refer to [2].

Acknowledgment: C.M. thanks the Netherlands Organization for Scientific Research (NWO) for a Ph.D. grant within the program on Computational Science.
[1] E. van Veldhuizen, Electrical Discharges for Environmental Purposes: Fundamentals and Applications (Nova Science Publishers, 2000).

[2] U. Ebert, C. Montijn, T. Briels, W. Hundsdorfer, B. Meulenbroek, A. Rocco, and E. van Veldhuizen (2006), http://www.cwi.nl/ftp/CWIreports/MAS/MASE0529.pdf, to appear in Plasma Source Sci. Technol.

[3] E. Bazelyan and Y. Raizer, Lightning Physics and Lightning Protection (IOP Publishing, Bristol, U.K., 2000).

[4] D. Sentman, E. Wescott, D. Osborne, and M. Heavner, Geophys. Res. Lett. 22, 1205 (1995).

[5] V. Pasko and H. Stenbaek-Nielsen, Geophys. Res. Lett. 29, 82(1 (2002).

[6] E. Gerken, U. Inan, and C. Barrington-Leigh, Geophys. Res. Lett. 27, 2637 (2000).

[7] H. Raether, Z. Phys. 112, 464 (1939).

[8] L. Loeb and J. Meek, J. Appl. Phys. 11, 438 (1940).

[9] S. Dhali and P. Williams, J. Appl. Phys. 62, 4696 (1987).

[10] P.A. Vitello, B.M. Penetrante, and J.N. Bardsley, Phys. Rev. E 49, 5574 (1994).

[11] A. Kulikovsky, J. Phys. D: Appl. Phys. 30, 441 (1997).

[12] S. Pancheshnyi, S. Starikovskaia, and A. Starikovskii, J. Phys. D: Appl. Phys. 34, 105 (2001).

[13] P.A. Vitello, B.M. Penetrante, and J. Bardsley, Nonthermal Plasma Techniques for Pollution Control, NATO ASI Ser., Ser. G 34(A), 249 (1993).
[14] A. Hallac, G. Georghiou, and A. Metaxas, J. Phys. D: Appl. Phys. 36, 2498 (2003).

[15] G. Georghiou, A. Papadakis, R. Morrow, and A. Metaxas, J. Phys. D: Appl. Phys. 38, R303 (2005).

[16] M. Arrayás, U. Ebert, and W. Hundsdorfer, Phys. Rev. Lett. 88, 174502 (2002).

[17] A. Rocco, U. Ebert, and W. Hundsdorfer, Phys. Rev. E 66, 035102(R) (2002).

[18] N. Liu and V. Pasko, J. Geophys. Res. 109, A04301 (2004).

[19] A.A. Kulikovsky, Phys. Rev. Lett. 89, 229401 (2002).

[20] S. Pancheshnyi and A. Starikovskii, J. Phys. D: Appl. Phys. 36, 2683 (2003).

[21] B. Meulenbroek, A. Rocco, and U. Ebert, Phys. Rev. E 69, 067402 (2004).

[22] B. Meulenbroek, U. Ebert, and L. Schäfer, Phys. Rev. Lett. 95, 195004 (2005).

[23] U. Ebert and W. Hundsdorfer, Phys. Rev. Lett. 89, 229402 (2002).

[24] U. Ebert, W. van Saarloos, and C. Caroli, Phys. Rev. E 55, 1530 (1997).

[25] C. Montijn, W. Hundsdorfer, and U. Ebert (2006), http://arxiv.org/abs/physics/0603070

[26] U. Ebert and W. van Saarloos, Physica D 146, 1 (2000).

[27] J. Wackers, J. Comp. Appl. Math. 180, 1 (2005). 